\title{
Revamped accelerator seeks quark's secrets
}

Geoff Brumfiel, Washington

An ageing particle accelerator in New York state is to get a fresh lease of life - hunting down the mysteries of the charm quark, one of the most elusive fundamental buildingblocks of matter.

The US National Science Foundation will give Cornell University's particle-physics laboratory \$124 million over five years to pursue the investigation, and to upgrade the synchrotron light source attached to the particle accelerator.

The Cornell Electron Storage Ring (CESR), which sits under a sports field on the university's Ithaca campus, was built in 1979 to examine collisions between electrons and positrons, their antimatter counterparts. For most of the facility's life, high-

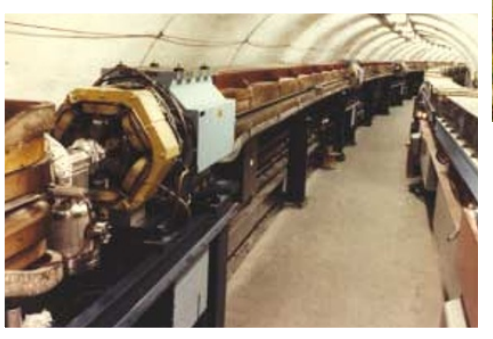

- the right energy, researchers hope, to generate charm quarks.

Charm quarks, which are among the six quarks featured in physicists' standard model, were first observed in 1974 by teams led by Burt Richter, at the Stanford Linear Accelerator Center (SLAC) in California, and Samuel Ting at Brookhaven National Laboratory in New York state. But according to Cassel, "there are a number of crucial things we still don't know about them". For example, researchers have still to obtain precise measurements of the lifetimes of charm-filled particles and the various ways in which they decay.
The revamped facility will also study the strong nuclear force, which binds quarks together to create protons and neutrons, and also keeps those particles together to form atomic nuclei, despite the huge repulsive electromagnetic force between them. In particular, physicists will be searching for 'glueballs', which are clusters of gluons, the particles that carry the strong force. "All theories of the strong force demand that there be glueballs," says Cassel, "but nobody has been able to verify that they exist."

"It's a superb team and the timing is just right," says Persis Drell, a former deputy director of Cornell's particle-physics lab and now associate director for research at SLAC. Drell says that the new investigation will neatly complement Stanford's work on particles known as B-mesons. Many researchers hope that studies of B-mesons will unravel the mystery of why there is more matter than antimatter in the Universe (see Nature 419, 24-27; 2002). "When a B-meson decays, it decays into a charm quark most of the time," Drell explains. "So these measurements will be of value to our experiments."

Some \$25 million of the CESR's new funding will go on upgrading its synchrotron light source, known as the Cornell High Energy Synchrotron Source. The source produces $\mathrm{X}$-rays with which visiting researchers can probe materials and molecules. But because Cornell's particle physicists now plan to run the accelerator at energies that are too low to produce X-rays, they will have to work at different times from the visiting scientists. glers', which act like speed bumps to slow the electrons and positrons down to $2.0-2.5 \mathrm{GeV}$

\section{NATO reform promises more publicity for less science}

Quirin Schiermeier, Munich

The North Atlantic Treaty Organisation (NATO) is to cut back its small but highly respected Science Programme and merge it with its public-relations office.

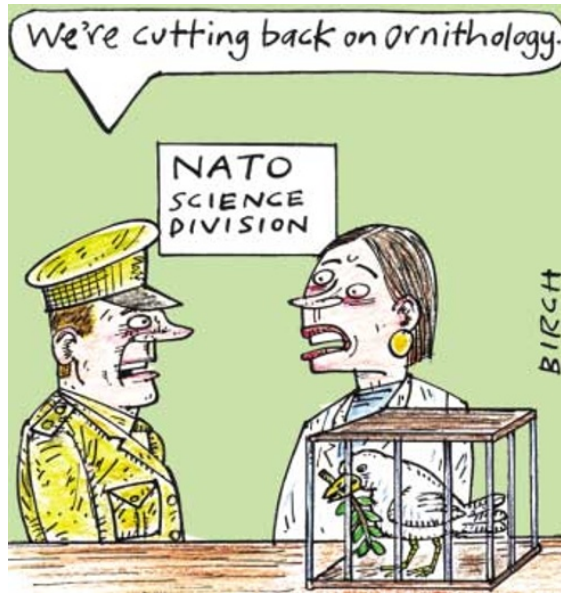

NATO's science division, which was set up in 1958, has supported high-quality military and civilian research. Its goal has been to strengthen scientific links between NATO states, and since the end of the cold war it has concentrated on supporting partnerships with scientists in new member states in central Europe and the 22 NATO 'partner countries' in eastern Europe and mid-Asia.

But the programme's 2003 budget has been cut to US\$20 million, from $\$ 23$ million last year. And next year it will be merged with NATO's Office of Information and Press to form a new public diplomacy division.

The move worries some researchers, who fear the programme may be heading for oblivion. "The situation seems stable for the coming year, but I am not very optimistic about the longer-term future of the Science Programme," says Charles
Buys, a geneticist at the University of Groningen who represents the Netherlands on NATO's Science Committee, which was informed of the planned change when it met in Brussels on 6 March.

But Brian Heap, who represents Britain on the committee, says that the new structure could actually raise the profile of NATO's research. "It is crucial to bring to public attention the fact that NATO is promoting peace and solidarity, while tackling the humanitarian challenges in its partner countries," he says.

NATO plans to continue supporting partnerships that involve researchers in the new member states, but they will be reduced in number and limited to research topics directly relevant to NATO's mission, such as counter-terrorism. www.nato.int/science 